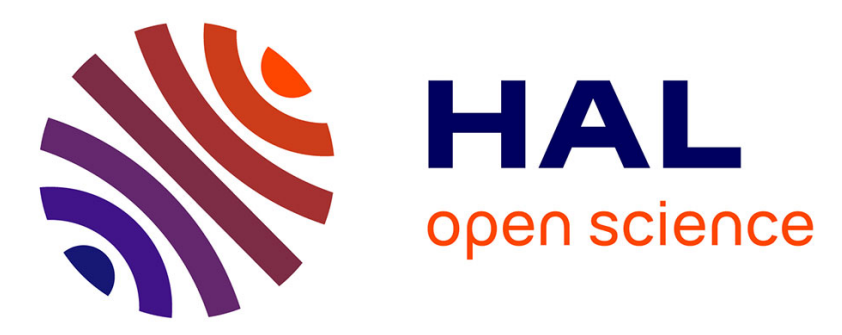

\title{
Multipass cells: 1D numerical model and investigation of spatio-spectral couplings at high nonlinearity
}

Nour Daher, Florent Guichard, Spencer W. Jolly, Xavier Delen, Fabien Quere, Marc Hanna, Patrick Georges

\section{- To cite this version:}

Nour Daher, Florent Guichard, Spencer W. Jolly, Xavier Delen, Fabien Quere, et al.. Multipass cells: 1D numerical model and investigation of spatio-spectral couplings at high nonlinearity. Journal of the Optical Society of America B, 2020, 37 (4), pp.993-999. 10.1364/JOSAB.386049 . hal-02566460

\section{HAL Id: hal-02566460 \\ https://hal-iogs.archives-ouvertes.fr/hal-02566460}

Submitted on 13 May 2020

HAL is a multi-disciplinary open access archive for the deposit and dissemination of scientific research documents, whether they are published or not. The documents may come from teaching and research institutions in France or abroad, or from public or private research centers.
L'archive ouverte pluridisciplinaire $\mathbf{H A L}$, est destinée au dépôt et à la diffusion de documents scientifiques de niveau recherche, publiés ou non, émanant des établissements d'enseignement et de recherche français ou étrangers, des laboratoires publics ou privés. 


\title{
Multipass cells: 1D numerical model and investigation of spatio-spectral couplings at high nonlinearity
}

\author{
Nour Daher, ${ }^{1},{ }^{*}$ Florent Guichard,${ }^{2}$ Spencer Jolly,${ }^{3}$ Xavier Delen, ${ }^{1}$ Fabien Quere, ${ }^{3}$ \\ Marc Hanna, ${ }^{1}$ AND Patrick GeORGES ${ }^{1}$ \\ ${ }^{1}$ Laboratoire Charles Fabry, UMR 8501, Institut d'Optique, CNRS, Université Paris Saclay, 2 Avenue Augustin Fresnel, 91127 \\ Palaiseau, France \\ ${ }^{2}$ Amplitude Laser, 11 Avenue de Canteranne, Cité de la Photonique, 33600 Pessac, France \\ ${ }^{3}$ LIDYL, CEA, CNRS, Université Paris-Saclay, CEA Saclay, 91191 Gif-sur-Yvette, France \\ *Corresponding author: nour.daher@institutoptique.fr
}

Received XX Month XXXX; revised XX Month, XXXX; accepted XX Month XXXX; posted XX Month XXXX (Doc. ID XXXXX); published XX Month XXXX

\begin{abstract}
Multipass cells (MPC) are used nowadays as nonlinear tools to perform spectral broadening and temporal manipulation of laser pulses while maintaining a good spatial quality and spatio-spectral homogeneity. However, intensive 3D nonlinear spatio-temporal simulations are required to fully capture the physics associated to pulse propagation inside these systems. In addition, the limitations of such scheme are still under investigation. In this study, we first establish a 1D model as a useful design tool to predict the temporal and spectral properties of the output pulse for nearly Gaussian beams, in a wide range of cavity configurations and nonlinearity levels. This model allows to drastically reduce the computation time associated to MPC design. The validity of the 1D model is first checked by comparing it to 3D simulations. The results of the 1D model are then compared with experimental data collected from a near concentric gas-filled multipass cell presenting a high level of nonlinearity, enabling the observation of wavebreaking. In a second part, we experimentally characterize the spatio-spectral profile at the output of this experimental setup, both with an imaging spectrometer and with a complete 3D characterization method known as INSIGHT. The results show that gas-filled multipass cells can be used at peak power levels close to the critical power without inducing significant spatio-spectral couplings in intensity or phase.
\end{abstract}

http://dx.doi.org/10.1364/AO.99.099999

\section{INTRODUCTION}

Nonlinear optics in single mode fibers is a research area that has bloomed continuously since the 1980 's until today [1]. It has allowed both the observation and understanding of fundamental phenomena in nonlinear physics, as well as enabled a plethora of applications, for instance in telecommunications and coherent light sources. One of the reasons for the success of this field is the one dimensional nature of light propagation along the fiber. This simplifies the dynamics considerably, and makes it easier to grasp nonlinear phenomena that can still be of great complexity. One of the salient examples is supercontinuum generation, which is much easier to describe and predict in optical fibers [2] than in bulk media [3].

In terms of applications, one of the weaknesses of single mode optical fibers is that the energy or peak power of pulses that can be manipulated using nonlinear optics phenomena is limited by the small transverse dimensions of the fiber core.
In silica core fibers, self-focusing limits the peak power to values in the range $1-10 \mathrm{MW}$. Hollow core fibers allow to increase this value [4], but in all cases there is a small but nonzero overlap between the guiding material and the optical field, which makes energy scaling difficult in practice. The most robust solution to scale the energy while retaining a guided propagation is to use hollow capillaries [5]. These structures are lossy and multimode by nature, but can approximate a low loss single mode waveguide by proper excitation of the fundamental mode and by selecting a large enough core diameter.

Recently, multipass cells (MPC) either including a bulk medium [6] or filled with a rare gas [7-9] have been used to perform nonlinear temporal compression of femtosecond pulses in various regimes (normal dispersion [6-9], soliton compression in anomalous dispersion [10]), in a large range of pulses energies (from $\mu \mathrm{J}[6,11]$ to $\mathrm{mJ}[7,9]$ ), and output pulse durations (from $>100$ fs [6] to few cycles $[10,12]$ ). In 
all these demonstrations, it was observed that the MPC essentially acts like a 1D nonlinear object, with negligible impact of the nonlinearity on the spatial properties of the beams. MPCs can therefore be seen as a vehicle to perform 1D nonlinear functions or experiments with energetic pulses.
They provide a large number of design degrees of freedom in terms of nonlinearity (bulk plates or gas with adjustable pressures) and dispersion engineering (through the mirror coatings). They can also provide long propagation distances in the nonlinear medium with a compact setup.

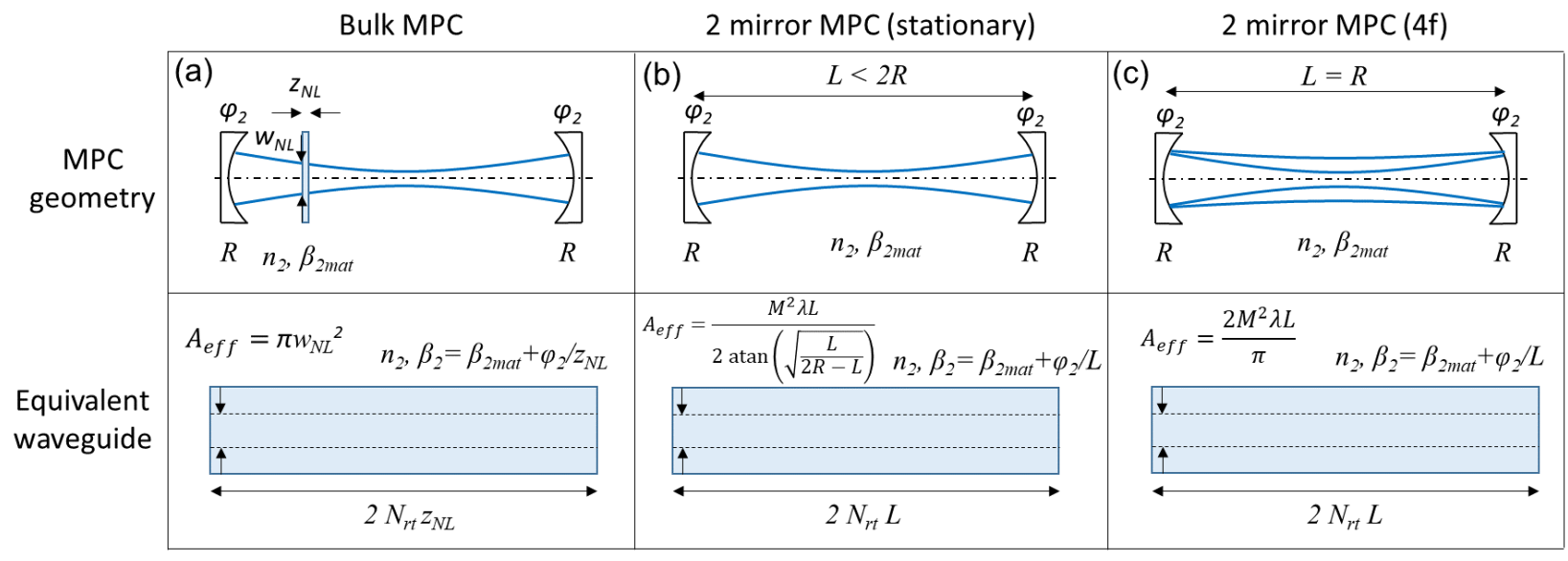

Fig. 1. Summary of equivalent waveguide parameters for three MPC geometries: bulk medium (a), stationary gas-filled MPC (b), and 4f gas-filled MPC (c).

In this article we address two questions. First, we aim at assessing to which degree a MPC can be thought of as a simple 1D nonlinear waveguide. We first introduce the notion of "equivalent waveguide" for different types of MPCs, and compare numerically a full $3 \mathrm{D}$ propagation model to the simplified 1D model obtained using this notion. The developed 1D model is shown to quantitatively predict the observed temporal and spectral properties of the output pulse when the nonlinearity level is low enough to essentially preserve the beam Gaussian shape and caustic evolution. The 1D model is then compared with an experiment in a near concentric MPC at increasing nonlinearity level, and is shown to remain valid at peak power values above half the critical power. In particular, it is able to reproduce the observation of wavebreaking, a well-known phenomenon in the context of nonlinear fiber optics in normal dispersion regime.

In a second part, we experimentally study the spatiospectral profile at the output of MPCs as the peak power approaches the critical power. This is done using both an imaging spectrometer and a complete 3D characterization technique known as INSIGHT [13]. The results show that the output pulses are essentially free of spatio-temporal couplings even at peak power levels close to the critical power.

\section{EQUIVALENT WAVEGUIDE AND 1D MODEL}

We start by defining the equivalent 1D model for different MPC geometries. We assume a Gaussian beam shape and propagation in order to establish this model. Since linear effects such as dispersion or attenuation are not affected by the changes in beam size, they can be directly transposed from the $3 \mathrm{D}$ situation to the $1 \mathrm{D}$ situation. We therefore focus on the nonlinear effects.
In the case of an MPC including a thin plate of bulk medium, as shown in Fig. 1(a), the simplest approach is to neglect the nonlinearity introduced by propagation outside of the medium. The beam size inside the material can be considered constant if its thickness is much less than the Rayleigh range. In this case, the equivalent waveguide is simply a waveguide with an effective area equal to $\pi w_{N L}{ }^{2}$, where $w_{N L}$ is the beam waist in the nonlinear medium, a length given by $2 N_{r t} z_{N L}$, where $N_{r t}$ is the number of roundtrips and $z_{N L}$ is the thickness of the medium, and the nonlinear index $n_{2}$ is the same as the bulk material. The dispersive properties of the MPC mirrors can be either accounted for as discrete elements inserted after each propagation length $z_{N L}$ to mimic the real implementation, or distributed over the propagation inside the medium. The use of such a "bulk" MPC allows peak powers in excess of the critical power, and equivalent propagation in a piece of bulk material longer than the selffocusing distance. Given that the nonlinearity of gas is typically three orders of magnitude less than that of bulk media, this equivalent 1D approach is reasonable, as an example, for cells of lengths in the few tens of centimeters range, and nonlinear media length in the few $\mathrm{mm}$ range, in which case the nonlinearity accumulated in the medium accounts for approximately $90 \%$ of the total.

Let us now consider the case of a symmetric two-mirror Herriot cell [14], with mirror radius of curvature $R$ and distance between the mirrors $L$, filled with a gas with nonlinear index $n_{2}$ and dispersion $\beta_{2}$, as depicted in Fig. 1(b). By identifying the nonlinearity accumulated over one pass [15] and the nonlinearity accumulated in a waveguide with a constant beam size, the equivalent waveguide is found to have the following properties: same overall length $2 N_{r t} L$, same nonlinear index $n_{2}$, same dispersion $\beta_{2}$ if the mirror dispersion 
is added as an additional discrete contribution at each propagation length $L$, and an effective area given by

$$
\boldsymbol{A}_{\text {eff }}=\boldsymbol{M}^{2} \frac{\lambda L}{2 \operatorname{atan}\left(\sqrt{\frac{L}{2 R-L}}\right)},
$$

Where $\lambda$ is the central wavelength, and $M^{2}$ is the beam quality parameter of the input beam.

Finally, we consider the case of a MPC in a $4 \mathrm{f}$ configuration, shown in Fig. 1(c), as used in [7]. This geometry allows an additional degree of freedom in the design, since the smallest beam waist size in the gas is now unrelated to the mirror radius of curvature and cell length. In this case, using the same approach as in [15], the $B$-integral (averaged over the beam transverse profile) accumulated over one roundtrip is found to be given by

$$
B=\frac{P_{\text {peak }}}{M^{2} P_{\text {crit }}} \frac{\pi^{2}}{4},
$$

where $P_{\text {peak }}$ is the pulse peak power and $P_{\text {crit }}=\lambda^{2} / 8 n_{2}$ is the critical power for self-focusing in the gas medium. One remarkable result is that this $B$-integral does not depend on the chosen small waist, because the large waist size is inversely proportional to the small one. As a consequence the equivalent waveguide effective area is given by

$$
A_{\text {eff }}=M^{2} \frac{2 \lambda L}{\pi}
$$

Figure 1 summarizes the equivalent waveguide characteristics in these three MPC geometries. We now compare numerical results obtained with a full $3 \mathrm{D}$ model of the propagation to this simplified $1 \mathrm{D}$ approach, to investigate its validity range.
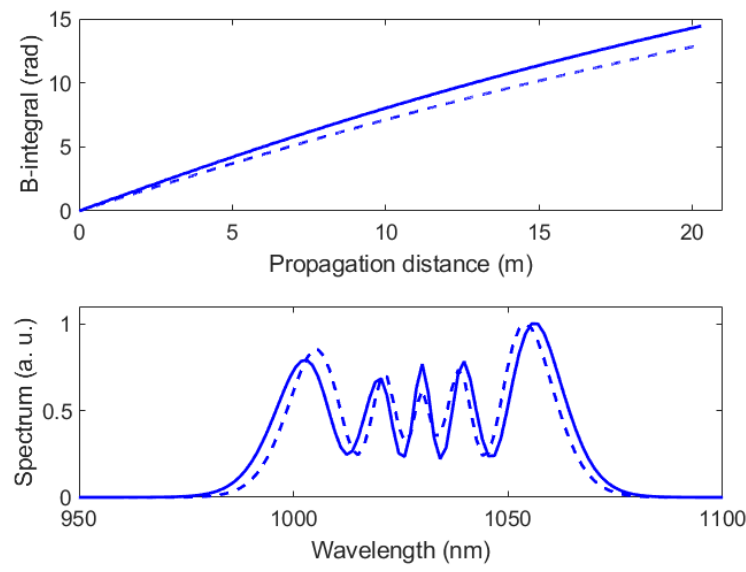

Fig. 2. Top-B-integral as a function of distance in the near confocal MPC for a xenon pressure of 1 bar. Bottom - Spectrum at the output of the MPC. The solid lines correspond the 3D simulation and the dashed lines to the $1 \mathrm{D}$ simulation.

The 3D model is described in [15] and is based on an envelope equation [16] solved using the split-step method. This model includes self-steepening in addition to diffraction, dispersion to all orders, and instantaneous Kerr effect. The 1D model is a standard split-step algorithm typically used to model propagation in single mode fibers and includes the exact same effects, excepting diffraction.

We first compare the 3D and 1D models for a set of parameters resembling the experimental configuration reported in [8]. The MPC is close to confocal with a mirror radius of curvature of $300 \mathrm{~mm}$, and a cell length of $290 \mathrm{~mm}$ filled with xenon at a pressure of one bar. The dispersion data is extrapolated from the Sellmeier coefficients reported in [17], and the nonlinear index is given by $n_{2}=5.2 \times 10^{-23} \mathrm{~m}^{2} / \mathrm{W}$ [18]. The input pulses have a Gaussian profile in time and space with a $300 \mathrm{fs}$ pulse duration and $150 \mu \mathrm{J}$ energy, and propagate along 35 roundtrips in the MPC. The input spatial profile is chosen to match the stationary condition in the MPC. These parameters correspond to a $P_{\text {peak }} / P_{\text {crit }}$ ratio of 0.18 . The $3 \mathrm{D}$ model is implemented on a grid size of $64 \times 64 \times 256$ in the space/time domain with steps $d x=d y=30 \mu \mathrm{m}, d t=10 \mathrm{fs}$, and $d z=12 \mathrm{~mm}$ in the propagation direction. For the 1D model, the equivalent effective area is $A_{\text {eff }}=0.19 \mathrm{~mm}^{2}$. Figure 2 shows the result of both models, clearly illustrating the fact that at this nonlinearity level, the 1D model quantitatively reproduces the results of the $3 \mathrm{D}$ model at a much reduced computational cost. This makes it a very useful design tool. The small discrepancy shows that the 1D model tends to slightly underestimate the spectral broadening, which is further explored in the following.
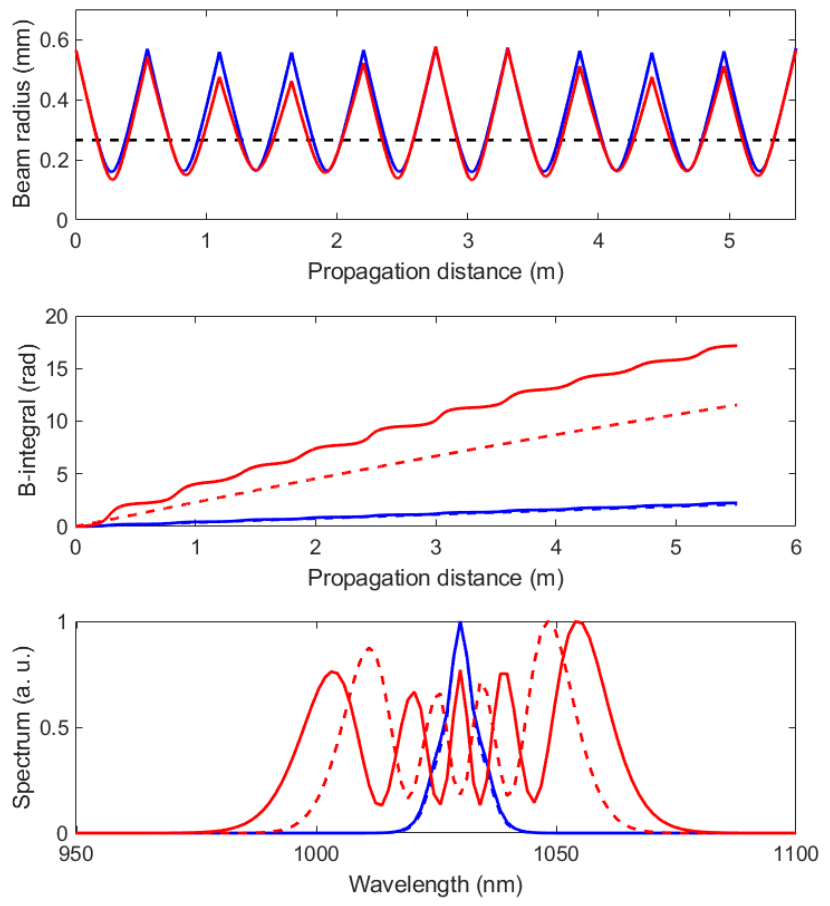

Fig. 3. Top - beam radius as a function of distance in the near concentric MPC for a xenon pressure of 0.5 bar (blue) and 3 bar (red). The black dashed line corresponds to the equivalent waveguide beam radius. Middle - B-integral as a function of distance in the MPC for a xenon pressure of 0.5 bar (blue) and 3 bar (red). The solid line is the 3D simulation and the dashed line is the 1D simulation. Bottom - Spectrum at the output of the MPC for a xenon pressure of 0.5 bar (blue) and $3 \mathrm{bar}$ (red). The solid line is the 3D simulation and the dashed line is the 1D simulation.

For the second comparison, we choose a xenon-filled cell with the following geometry: mirror radius of curvature 300 $\mathrm{mm}$, cell length $551 \mathrm{~mm}$, close to the concentric configuration. These dimensions were chosen to match the 
experimental parameters used in the next sections, and because the concentric configuration allows the use of relatively compact setups while keeping the beam size at the mirror large, which is important to avoid optical damage.

The input pulses have a Gaussian profile in time and space with a 350 fs pulse duration and $200 \mu \mathrm{J}$ energy, and propagate along 5 roundtrips in the MPC. This number of roundtrips is chosen to easily illustrate the comparison between the 3D and 1D models in terms of caustic evolution. Two xenon pressures are considered: 0.5 bar, corresponding to a low level of nonlinearity $\left(P_{\text {peak }} / P_{\text {crit }}=0.1\right)$, and $3 \mathrm{bar}$, corresponding to a very high level of nonlinearity $\left(P_{\text {peak }} / P_{\text {crit }}=0.6\right)$. The 3D model is implemented on a grid size of $128 \times 128 \times 256$ in the space/time domain with steps $d x=d y=50 \mu \mathrm{m}, d t=10 \mathrm{fs}$, and $d z=5.6 \mathrm{~mm}$ in the propagation direction. For the 1D model, the equivalent effective area is $A_{\text {eff }}=0.22 \mathrm{~mm}^{2}$. Figure 3 shows the comparison results. At low nonlinearity, the 1D model still follows closely the $3 \mathrm{D}$ model. The main reason is that the caustic evolution during propagation is essentially unmodified by the spatial Kerr effect, as shown in Fig. 3 (top). However, at high nonlinearity, the Kerr lens clearly modifies the beam caustic from the stationary situation. In particular, the first pass shows a beam waist smaller than in the linear case, and an oscillation of the beam size with respect to the unperturbed evolution is observed. This result in a higher level of nonlinearity than the 1D model predicts, as shown in Fig. 2 (middle). The output spectrum is therefore broader for the 3D model than for the 1D model. Note that these discrepancies appear at levels of nonlinearity that are higher than most experimental reports, making the 1D approximation still very useful as a design tool.

Although the beam sizes are modified by the spatial Kerr effect at high nonlinearity level, the 3D simulation shows no spatio-spectral intensity couplings at 3 bar of xenon pressure, corresponding to $P_{\text {peak }} / P_{\text {crit }}=0.6$. In this case the B-integral per roundtrip calculated assuming an unperturbed caustic and perfect homogenization is $2.5 \mathrm{rad}$, and the B-integral of the first roundtrip from the 3D simulation is $4.3 \mathrm{rad}$. This $3 \mathrm{D} \mathrm{B}-$ integral is calculated by taking the on-axis value divided by 2 , which should be equal to the average value of the 1D model in the case of perfect Gaussian beam and homogenization. Although it might be surprising that this very large nonlinearity does not induce significant spatio-spectral couplings [19], it has also been observed experimentally that a B-integral per roundtrip of $2.4 \mathrm{rad}$ does not result in the onset of spatio-spectral couplings in intensity, as revealed by an imaging spectrometer characterization [9]. We would like to point out that this is in contradiction with the result reported at the end of our earlier report [15] that showed a rapid increase of the spatio-spectral coupling for the concentric configuration as the nonlinearity is increased. After reexamination of the corresponding simulation data we believe this was the result of insufficient sampling density, a problem that is increasingly challenging as we approach the concentric configuration. Indeed, the beam divergence and size that must be accommodated by the sampling grid both increase. This leaves the question open of how much nonlinearity can be tolerated in a MPC before spatio-spectral couplings appear. We investigate this question experimentally in section 4.

\section{EXPERIMENTAL VALIDATION OF THE 1D MODEL}

We start by comparing the $1 \mathrm{D}$ model described above with an experiment designed to be able to observe wavebreaking. It is a phenomenon that is well-known in optical fibers [20], has been recently observed in MPCs [7], and requires a high level of nonlinearity, thereby providing an appropriate physical testbed for the 1D model described here. The experimental setup used in this study, shown in Fig. 4, includes a MPC that consists of two concave mirrors with a radius of curvature of $300 \mathrm{~mm}$ placed in a 3.8-bar xenon-filled gas chamber. The input laser pulse sent into the multipass cell originates from an Yb-doped fiber amplifier delivering a maximum input pulse of $200 \mu \mathrm{J}$ energy at a central wavelength of $1030 \mathrm{~nm}$ at $150 \mathrm{kHz}$ repetition rate, corresponding to a maximum average power of $30 \mathrm{~W}$. The measured values of the spatial beam quality $\mathrm{M}^{2}$ are $1.2 \times 1.2$ along two orthogonal axes.

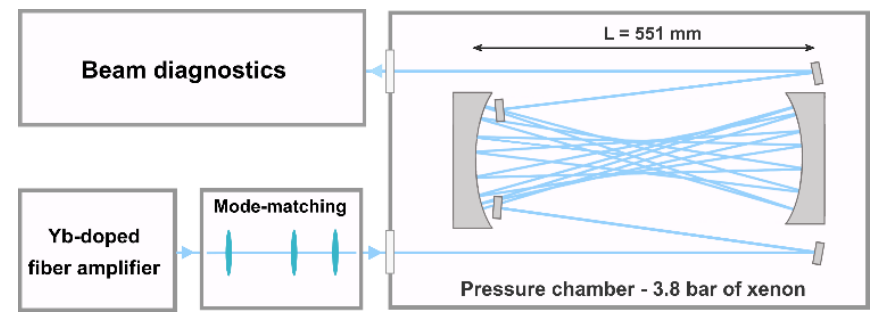

Fig. 4. Experimental setup.

Xenon was chosen as nonlinear medium due to its high nonlinear index among noble gases. At our gas pressure of 3.8 bar the nonlinear index is given by $n_{2}=2.0 \times 10^{-22} \mathrm{~m}^{2} / \mathrm{W}$, corresponding to a critical power for self-focusing of 0.67 $\mathrm{GW}$, and the group velocity dispersion is $296 \mathrm{fs}^{2} / \mathrm{m}$. This critical power is higher than yet very close to the maximum input peak power that can be reached by our laser source of $P_{\text {peak }}=0.59 \mathrm{GW}$, allowing us to investigate highly nonlinear regimes in this MPC. Two rectangular $3 \times 10 \mathrm{~mm}^{2}$ mirrors located in front of one of the MPC mirrors were used for inout coupling of the laser beam. The concave MPC mirrors are chosen to present a high reflection coefficient (>99.9\%) on a 980-1080 nm bandwidth and are coated with a dispersive HR coating with GDD $=-25 \pm 10 \mathrm{fs}^{2}$ that partially compensates the normal dispersion introduced by the nonlinear gas medium. The distance separating the two mirrors is chosen to be $551 \mathrm{~mm}$, near a concentric geometrical configuration. By finely adjusting the length between the two mirrors and the input coupling into the cell, we obtain 21 roundtrips resulting in a total propagation length of $24.5 \mathrm{~m}$ including the path before and after the MPC in the gas-filled enclosure. The beam is mode-matched using an arrangement of three lenses, in order to provide beam radii close to theoretical values of $154 \mu \mathrm{m}$ at the center of the cell expanding to $624 \mu \mathrm{m}$ on the concave MPC mirrors. This kind of MPC geometries is advantageous, as mentioned earlier, for inducing spectral broadening at relatively high input pulse energy while 
mitigating any damage of the MPC mirrors since the beam size becomes large on the mirrors allowing to maintain a fluence on the mirrors well below the damage threshold. However, with a relatively small waist at the center of the cell, one should pay attention to gas ionization. In our case, the maximum intensity at the center of the MPC is $7.9 \times 10^{11}$ $\mathrm{W} / \mathrm{cm}^{2}$, well below the ionization threshold for xenon.

The power transmission of the experimental setup is $86 \%$, including the mode matching optics. This reduced value compared to previous reports might be due to aging of the optical coatings. The beam exiting the MPC is collimated and sent to the diagnostics. The temporal characterization of the output pulse was made by means of a home-built Second Harmonic Generation Frequency Resolved Optical Gating (SHG-FROG) setup allowing us to investigate the occurrence of wave breaking for different values of input energies. This nonlinear effect is observed in nonlinear optical fibers when the combination of self-phase modulation (SPM) and groupvelocity dispersion (GVD) makes a pulse broaden and change its shape towards an almost rectangular form. This pulse evolution then features the appearance of fast temporal oscillations on both sides of the pulse [20]: the red-shifted light near the leading-edge travels faster for $\beta_{2}>0$ and overtakes the unshifted light in the tail of the pulse, while the opposite takes place at the trailing edge of the pulse. This gives birth to temporal interference oscillations and subsequent generation of new frequencies at the spectrum edge.

Fig. 5 shows the pulse temporal and spectral profile measured at different levels of input energy compared with the results obtained by the $1 \mathrm{D}$ numerical model described above, taking into account the measured spectrum and spectral phase of the input pulse. The temporal profile changes from a Gaussian-shaped pulse to a rectangularshaped pulse with oscillations appearing on both temporal edges of the pulse as the energy is increased. These oscillations are accompanied by the appearance of broad sidelobes on the pulse spectrum. Wave breaking is experimentally observed, in our case, at input energies $>60$ $\mu \mathrm{J}$. At $60 \mu \mathrm{J}$ input energy, the estimated B-integral at which wavebreaking occurs is $11.2 \mathrm{rad}$ [20], and the accumulated Bintegral obtained from the $1 \mathrm{D}$ model corresponding to the experimental parameters is $12 \mathrm{rad}$, in quantitative agreement. There is a good agreement between the experimental results and the model, at $P_{\text {peak }} / P_{\text {crit }}$ ratios up to $0.73(160 \mu \mathrm{J}$ pulse energy), illustrating the value of this simple $1 \mathrm{D}$ picture. While it might be surprising at first that the $1 \mathrm{D}$ model is still valid at such a high nonlinearity level, we believe that this is due to the fact that it is straightforward to account for an imperfect beam through the $\mathrm{M}^{2}$ value, as shown in section 2 . It is more difficult to do so with 3D models, since an exact shape of intensity and phase imperfections must be assumed or measured.
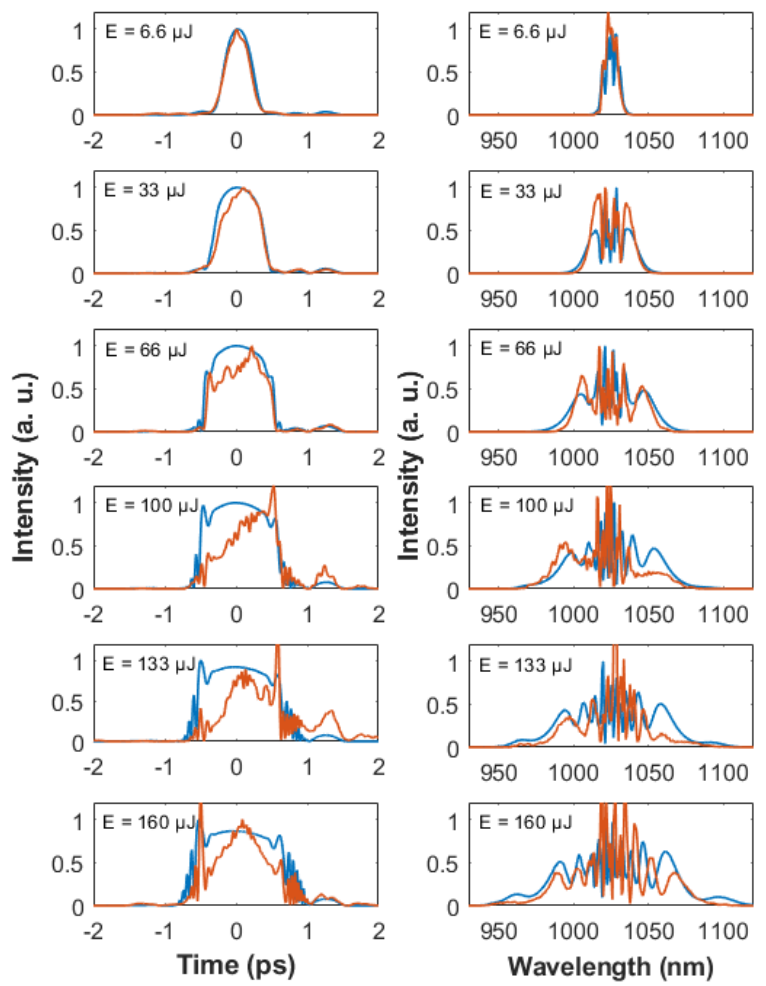

Fig. 5. Left column, FROG-retrieved temporal intensity profile (red) compared to numerically simulated temporal intensity profile (blue) obtained for different levels of input energy at the output of the MPC filled with 3.8 bar of xenon. Right column, measured spectrum (red) compared to spectrum obtained by simulation (blue).

\section{MEASUREMENT OF INTENSITY AND PHASE SPATIO-SPECTRAL PROFILES}

In this section, we investigate the spatio-spectral profiles at the output of the MPC as the nonlinearity increases. Indeed, although energy scaling limitations are well defined regarding gas ionization and mirror damage, the limitation in terms of nonlinearity (either in terms of B-integral per pass or peak power to critical power ratio) is not clear to our knowledge. In particular, it is not yet fully understood how far the nonlinearity level can be pushed in MPCs while still retaining a spatio-spectral couplings-free output beam, although a clear upper bound is the critical power in the gas medium. To study the spatio-spectral couplings in the beam after the multipass cell, we first use an imaging spectrometer setup. This setup is an f-f arrangement composed of an 800 lines/mm grating, a $50 \mathrm{~mm}$ focal length cylindrical lens and an InGaAs CCD camera with a $30 \mu \mathrm{m}$ pixel size. The camera is voluntarily saturated for the spectral components near $1030 \mathrm{~nm}$ in order to better observe the spatio-spectral structures of the full broadened spectrum. The results are plotted in Fig. 6 . 

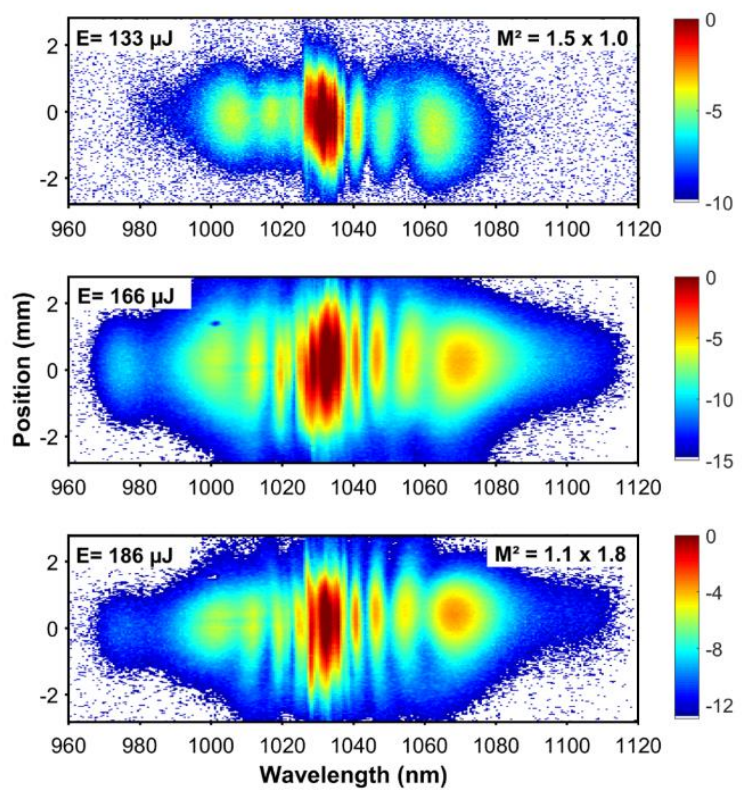

Fig. 6. Experimental spectro-imaging measurements in logarithmic (dB) scale for increasing energies of $133 \mu \mathrm{J}$ (top), $166 \mu \mathrm{J}$ (middle), and $186 \mu \mathrm{J}$ (bottom).

For $133 \mu \mathrm{J}$ input energy, corresponding to a $P_{\text {peak }} / P_{\text {crit }}$ ratio of 0.58 , the measured spectrum across the transverse profile does not show signs of spatio-spectral couplings. As the energy is increased to $166 \mu \mathrm{J}\left(P_{\text {peak }} / P_{\text {crit }}=0.73\right)$, some slight signs of couplings appear. At this point the beam pointing starts to be unstable. At $186 \mu \mathrm{J}$ pulse energy $\left(P_{\text {peak }} / P_{\text {crit }}=\right.$ $0.81)$ the beam is very unstable. However, when the spatiospectral profile is recorded using a low integration time, as shown at the bottom of Fig. 5, it is still not showing very clear signs of couplings in intensity. To quantify this assertion, the mean spectral overlap factor $\mathrm{V}$ [21] is $97 \%$ even at this extreme nonlinearity level. The mechanism for the observed beam instability is not clearly understood, but might be due to the fact that small initial fluctuations in intensity are transferred to changes in the beam propagation through the large amount of Kerr spatial phase.

In order to further asses the spatio-spectral properties of the broadened laser pulses, we use the INSIGHT technique [13], which involves spatially-resolved Fourier-transform interferometry at three positions around the focus. Using a phase retrieval algorithm this allows for reconstruction of the spatio-spectral phase in addition to the spectral amplitude already afforded by the Fourier-transform spectroscopy. The results of an INSIGHT measurement at $133 \mu \mathrm{J}$ can be seen in Fig. 7, showing both slices of intensity (a)-(c) and phase (d)(f) at three sample wavelengths, and reconstructed integrated quantities (g)-(i). INSIGHT characterization at higher energy levels was not possible because of the increasing shot-to-shot instability of the beam.

The power of the complete spatio-spectral measurement is the ability to measure both the amplitude and phase at all frequencies within the laser pulses. Therefore, the non- integrated processed data can provide information on frequency-resolved aberrations, the so-called spatio-temporal or spatio-spectral couplings [22]. Three maps of the spatial intensity and phase profiles at $1000 \mathrm{~nm}, 1030 \mathrm{~nm}$, and 1060 $\mathrm{nm}$ are shown in Fig. 6 (a)-(c) (intensity) and Fig. 6 (d)-(f) (phase). Although especially at $1060 \mathrm{~nm}$ the intensity profile begins to degrade, the phase maps contain very small magnitude low-order aberrations. In fact, in this case the main aberration is a minor wavelength-independent astigmatism, which does not contribute to spatio-temporal deterioration. We do not measure any low-order spatio-temporal couplings such as pulse-front tilt or pulse-front curvature. A single number, the so-called spatio-temporal Strehl ratio [23] can be used to assess the severity of the chromatic phase couplings. In the case of the data shown in Fig. 7, the spatio-temporal Strehl ratio is 0.9 , meaning that the spatio-temporal couplings (i.e. the purely chromatic aberrations which would not be possible to correct via a deformable mirror) are responsible for a decrease in focused intensity by $10 \%$.

Figure $7(\mathrm{~g})$ shows the spectrally-integrated beam profile, along with integrated 2D spectrograms in Fig. 7(h)-(i) that numerically reconstruct the imaging spectrometer used to produce the data in the top panel of Fig. 6. The integrated results provide a good benchmark for the INSIGHT results, in that they agree qualitatively with previous measurements. Despite the fact that fine features are not reproduced due to the limited spectral resolution of these INSIGHT measurements compared to the imaging spectrometer (related to the range of the delay scan done), the results, especially in Fig. 7(h)-(i), are comparable.

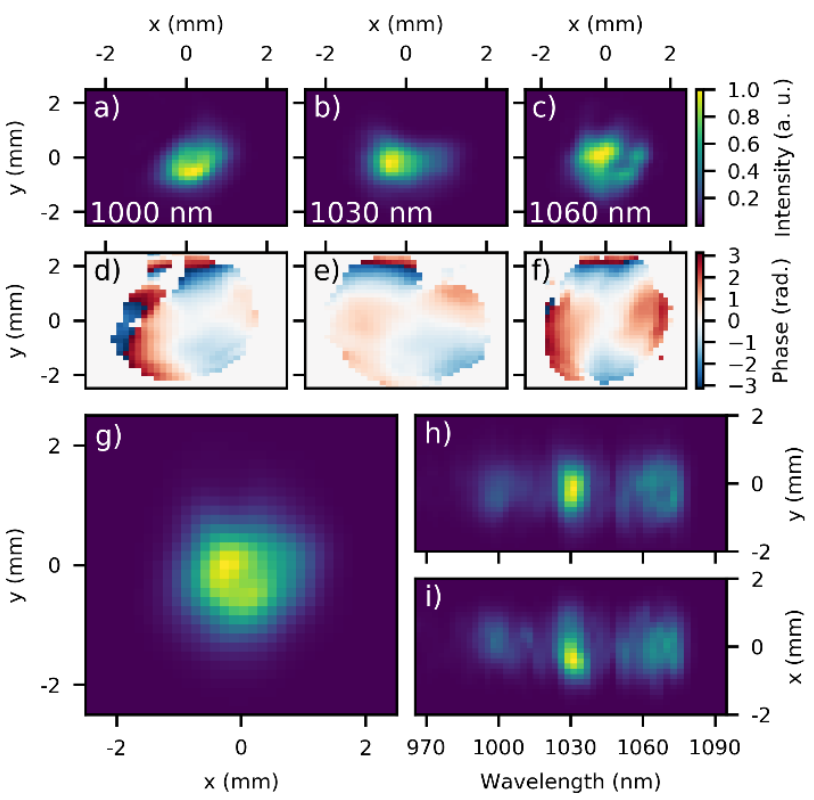

Fig. 7. Results of the INSIGHT measurement. The full spatio-spectral data from the INSIGHT measurement produces the 2D amplitude profiles (a-c) and phase profiles (d-f) for the three wavelengths shown of 1000,1030 , and $1060 \mathrm{~nm}$. The spectrally-integrated beam profile (g) and spatio-spectral profiles (h-i) provide a comparison to standard 
devices. In (h) and (i) the intensity is integrated along the spatial axis that is not shown.

\section{CONCLUSION}

To conclude, we have proposed a simple 1D numerical model that can be used to describe pulse propagation in MPCs with Gaussian beams, and is valid when the nonlinearity does not lead to major changes in the beam caustic along propagation. This model is tested experimentally in a near concentric xenon-filled MPC and allows to accurately reproduce the observation of wave breaking. We also perform a full 3D characterization of the electric field at the output of a MPC for the first time to our knowledge using the INSIGHT method. Despite the very high level of nonlinearity introduced, no severe spatio-spectral inhomogeneity at the output of the multipass cell has been observed, whether in intensity or phase. Our results show that MPCs can be used to perform nonlinear optical functions usually implemented in optical fibers while scaling the pulse energy.

\section{Funding Information.}

Agence Nationale de la Recherche (ANR) (ANR-10-LABX-0039-PALM, ANR-16-CE30-0027-01-HELLIX).

\section{REFERENCES}

1. G. P. Agrawal, "Nonlinear fiber optics," $5^{\text {th }}$ edition, Academic Press (2012).

2. J. M. Dudley, G. Genty, S. Cohen, "Supercontinuum generation in photonic crystal fiber," Rev. Mod. Phys. 78, 1135 (2006).

3. A. Dubietis, A. Couairon "Ultrafast supercontinuum generation in transparent solid-state media," Springer (2019).

4. T. Balciunas, C. Fourcade-Dutin, G. Fan, T. Witting, A. A. Voronin, A. M. Zheltikov, F. Gerome, G. G. Paulus, A. Baltuska, F. Benabid, “A strong-field driver in the single-cycle regime based on self-compression in a kagome fibre," Nat. Comm. 6, 6117 (2015).

5. M. Nisoli, S. De Silvestri, O. Svelto, R. Szipöcs, K. Ferencz, Ch. Spielmann, S. Sartania, and F. Krausz, "Compression of high-energy laser pulses below 5 fs," Opt. Lett. 22, 522-524 (1997).

6. Jan Schulte, Thomas Sartorius, Johannes Weitenberg, Andreas Vernaleken, and Peter Russbueldt, "Nonlinear pulse compression in a multi-pass cell," Opt. Lett. 41, 4511-4514 (2016).

7. M. Ueffing, S. Reiger, M. Kaumanns, V. Pervak, M. Trubetskov, T. Nubbemeyer, F. Krausz, "Nonlinear pulse compression in a gas-filled multipass cell," Opt. Lett. 43, 2070-2073 (2018).

8. L. Lavenu, M. Natile, F. Guichard, Y. Zaouter, X. Delen, M. Hanna, E. Mottay, and P. Georges, "Nonlinear pulse compression based on a gasfilled multipass cell," Opt. Lett. 43, 2252-2255 (2018).

9. M. Kaumanns, V. Pervak, D. Kormin, V. Leshchenko, A. Kessel, M. Ueffing, $\mathrm{Y}$. Chen, and T. Nubbemeyer, "Multipass spectral broadening of $18 \mathrm{~mJ}$ pulses compressible from 1.3 ps to $41 \mathrm{fs}$," Opt. Lett. 43 , 5877-5880 (2018).

10. G. Jargot, N. Daher, Loïc Lavenu, X. Delen, N. Forget, M. Hanna, and P. Georges, "Self-compression in a multipass cell," Opt. Lett. 43, 5643-5646 (2018).

11. J. Weitenberg, T. Saule, J. Schulte and P. Rußbüldt, "Nonlinear Pulse Compression to Sub-40 fs at $4.5 \mu$ Pulse Energy by Multi-Pass-Cell Spectral Broadening," in IEEE Journal of Quantum Electronics, vol. 53, no. 6, pp. 1-4, Dec. 2017, Art no. 8600204.

12. L. Lavenu, M. Natile, F. Guichard, X. Délen, M. Hanna, Y. Zaouter, and P. Georges, "High-power two-cycle ultrafast source based on hybrid nonlinear compression," Opt. Express 27, 1958-1967 (2019).
13. A. Borot and F. Quéré, "Spatio-spectral metrology at focus of ultrashort lasers: a phase-retrieval approach," Opt. Express 26, 26444-26461 (2018).

14. D. Herriott, H. Kogelnick, and R. Kompfner, "Off-axis paths in spherical mirror interferometers," Appl. Opt. 3, 523-526 (1964).

15. M. Hanna, X. Délen, L. Lavenu, F. Guichard, Y. Zaouter, F. Druon, and P. Georges, "Nonlinear temporal compression in multipass cells: theory," J. Opt. Soc. Am. B 34, 1340-1347 (2017).

16. T. Brabec and F. Krausz, "Nonlinear optical pulse propagation in the single-cycle regime", Phys. Rev. Lett. 78.3282(1997).

17. A. Bideau-Mehu, Y. Guern, R. Abjean, and A. Johannin-Gilles, "Measurement of refractive indices of neon, argon, krypton, and xenon in the 253.7-140.4 nm wavelength range. Dispersion relations and estimated oscillator strengths of the resonance lines", J. Quant. Spectrosc. Radiat. Transfer 25, 395-402 (1981)

18. J. K. Wahlstrand, Y.-H. Cheng, and H. M. Milchberg, "High Field Optical Nonlinearity and the Kramers-Kronig Relations", Phys. Rev. Lett. 109 (2012).

19. N. Milosevic, G. Tempea, and T. Brabec, "Optical pulse compression: bulk media versus hollow waveguides," Opt. Lett. 25, 672-674 (2000).

20. D. Anderson, M. Desaix, M. Lisak, and M. L. Quiroga-Teixeiro, "Wave breaking in nonlinear-optical fibers," J. Opt. Soc. Am. B 9, 1358-1361 (1992).

21. J. Weitenberg, A. Vernaleken, J. Schulte, A. Ozawa, T. Sartorius, V. Pervak, H. Hoffmann, T. Udem, P. Russbüldt, and T. W. Hänsch, "Multipass-cell-based nonlinear pulse compression to $115 \mathrm{fs}$ at $7.5 \mu$ pulse energy and $300 \mathrm{~W}$ average power," Opt. Express 25, 20502-20510 (2017).

22. S. Akturk, X. Gu, P. Bowlan, \& R. Trebino, "Spatio-temporal couplings in ultrashort laser pulses," Journal of Optics 9, 093001 (2010).

23. A. Jeandet, A. Borot, K. Nakamura, S. W. Jolly, A. J. Gonsalves, C. Tóth, H.S. Mao, W. P. Leemans, \& F. Quéré, "Spatio-temporal structure of a Petawatt femtosecond laser beam," Journal of Physics: Photonics 1 , 035001 (2019). 